\section{Vol. 70, No. 27}

In the report "Outcomes Among Patients Referred to Outpatient Rehabilitation Clinics After COVID-19 diagnosis - United States, January 2020-March 2021," on page 967, the following authors' names should have read, "Meredith G. Dixon, MD" and "Caitlyn Lutfy, MPH." On page 970, in Table 3, in the row for "Social participation ability," in the columns for "Post-COVID-19 patients," "Control patients," and "mean difference" the summary scale T-score mean standard deviations and mean differences should have read, "46.6 (44.7 to 48.6)," "50.5 (50.0 to 51.1)," and “ $-4.2(-6.4$ to -2.0$)$," respectively.

\section{Vol. 70, No. 30}

In the report "Progress Toward Hepatitis B Control World Health Organization European Region, 2016-2019," on page 1030, in the second column, first paragraph, the last sentence should have read, "Of the 21 countries with universal HepB-BD that reported birth dose coverage to WHO, ${ }^{\dagger \dagger \dagger}$ coverage with timely HepB-BD during 2016-2019 was $\geq 90 \%$ in 19-20 (90\%-95\%) countries." 